\title{
THE INFLUENCE OF CAFFEINE ON SPORTS PERFORMANCE
}

Teodora DOMINTEANU ${ }^{1}$

\begin{abstract}
Caffeine is the most used substance in the world as a tonic of the nervous system. It is found in a whole range of beverages, foods, and pharmaceuticals including coffee, tea, sour drinks, energy drinks, chocolate, aspirin and even in some shampoos and soaps. The US Food and Drug Administration (FDA) calls caffeine "a multi-purpose food substance generally recognized as safe." The most important source of caffeine in the world is coffee consumption.
\end{abstract}

Keywords: caffeine, effects, sports performance.

JEL Classification: I1, I12

DOI: 10.24818/mrt.20.11.02.01

\section{Introduction}

Caffeine is a bitter, crystalline white alkaloid with a strong tonic effect on the central nervous system and metabolism. The stimulant effect of caffeine can temporarily dim the feeling of dizziness by amplifying the state of attention, the power of concentration and the speed of reaction as well as by increasing the production of energy and by decreasing the level of perception of the effort during the training. Caffeine is generally consumed in the form of drinks but can be consumed in larger quantities and in the form of capsules or chewing gum.

The rate of caffeine absorption in the bloodstream varies depending on how it is ingested. Caffeine consumed in liquid form (coffee, tea, cola) penetrates the stomach quickly and generally reaches the bloodstream and tissues within 45 minutes. Caffeine from chewing gum does not pass through the stomach and reaches the bloodstream faster than that from drinks, through the mouth mucosa, its effects can be felt even in 10 minutes. For athletes who need a quick energy boost before a competition or training, caffeine consumption in the form of chewing gum may be more effective than in the

\footnotetext{
${ }^{1}$ Professor Ph.D., Academy of Economic Studies, Department of Physical Education and Sport, E-mail: tdominteanu77@gmail.com
} 
form of beverages, but they should be aware of its side effects if not are used to caffeine in this form.

Half the lifetime of caffeine, more precisely the time needed to metabolize half the amount of caffeine in the body is generally considered to be 5 hours. However, the rate at which caffeine is metabolized by the liver may be influenced by multiple factors, such as age, liver function, certain medication, pregnancy, and the level of liver enzymes. For women who are taking contraceptives or are pregnant, the half-life of caffeine can reach up to 10 hours $^{36}$.

For athletes, it is important to know how these factors influence caffeine metabolism. In athletes, excessive and reckless consumption of caffeine can have paradoxical effects of decreasing physical and mental performance.

In recent years there have been a lot of studies on caffeine and it has been proven that it can be very effective in increasing energy production in athletes. Studies have shown an increase in the endurance (endurance) of athletes between $7 \%$ and $51 \%$. The rate of increase in performance is influenced by the dose of caffeine consumed ${ }^{36}$.

An amount of 3 to $9 \mathrm{mg}$ of caffeine per $\mathrm{kg}$ of body mass has been shown to have an increase in energy production. Doses lower or higher have been shown to have no benefit. On the contrary, some studies have shown that they can have a diminishing effect on physical and mental performance. It is therefore imperative for athletes who want to use caffeine as an energyproducing adjunct to consult with a sports nutritionist about a consumption protocol and to experiment with different doses, so as to determine the optimal level that can ensure their performance and reduction side effects ${ }^{36}$.

\section{Performance in sports}

According to research, caffeine can help improve physical performance during endurance and high-intensity exercises ${ }^{1-12}$. Studies have shown that during endurance physical exercises (eg, aerobic exercises in sports that last more than five minutes, such as running, cycling and boating), caffeine improves counterchronometer performance and may be associated with a reduction in muscle pain $^{3-12}$. Also, research suggests that caffeine can contribute positively during highintensity (anaerobic) short physical exercises, e.g. athletes who perform high intensity exercises and team sports ${ }^{13-19}$.

The European Food Safety Authority (EFSA) concluded that there is an association between caffeine consumption and an increase in endurance performance, endurance capacity and a reduction in calculated effort or physical exertion during exercise ${ }^{20}$. 
Although much of the research has been done among professional athletes, studies among sedentary and physically impaired individuals also suggest that caffeine intake may improve the performance of non-trained athletes ${ }^{21,22}$.

Caffeine can exert its effect through adenosine receptor antagonism in the brain - a pathway that leads to increased adrenaline production, which stimulates energy production and improves blood flow to muscles and heart ${ }^{10,11}$. Also, caffeine can modulate central fatigue, a type of exhaustion caused by neurochemical changes in the brain associated with prolonged exercise and can, in turn, influence the degree of perceived effort, perceived pain and vigor, all leading most likely to improve the performance ${ }^{11,12}$.

Caffeine is widely defined as an ergogenic stimulant, more precisely, a substance that enhances the ability to work or exercise. In 1978, Costill and his colleagues were the first to demonstrate that $330 \mathrm{mg}$ of caffeine administered one hour before exercise at maximum oxygen consumption of $80 \%$ on an ergometer bicycle increases the time to exhaustion ${ }^{33}$.

Research suggests that performance benefits may be visible in the case of moderate caffeine consumption (approximately 3mg / kg body weight or 200 - 300 mg caffeine) in several sports, including endurance, stop-and-go events such as sports. team with rocket and activities sustained by high intensity such as swimming and boating ${ }^{1,2}$.

In 2015, the European Food Safety Authority (EFSA) published the Scientific Opinion on Coffee Safety, concluding that 'consumption of caffeine in single doses up to 200mg (almost $3 \mathrm{mg}$ / kg body weight) from all sources does not raise concerns with regarding the safety of the adult population in general, even when consumed less than two hours before performing intense physical exercise under normal environmental conditions ${ }^{32}$.

An analysis paper from 2009 focused on performance in endurance activities with a duration of more than five minutes, measuring running time, cycling or boating on a set distance, compared to the time to exhaustion, which better reflects the conditions. of typical competition. The inclusion criteria were met by 21 papers covering 33 studies $^{3}$. Thirty of them showed an improvement in the average performance of $3.2 \pm 4.3 \%$ due to caffeine consumption. The analysis concluded that caffeine ingestion can be an effective ergogenic aid for endurance athletes when consumed in moderate amounts (3-6 mg / kg body weight), before and / or during exercise ${ }^{3}$. However, refraining from caffeine at least 7 days before an event optimized the ergogenic effect of caffeine on performance within the event ${ }^{3}$.

In 2011, a study examining caffeine withdrawal and high-intensity endurance cycling performance suggested that a $3 \mathrm{mg} / \mathrm{kg}$ body caffeine intake significantly improved sports performance, regardless of whether regular caffeine users were required to abstain. for 4 days ${ }^{4}$.

Other research published in 2012 concluded that a $3 \mathrm{mg} / \mathrm{kg}$ body caffeine intake seems to improve cycling performance; although doubling the consumption 
to $(6 \mathrm{mg} / \mathrm{kg}$ body weight) did not confer a further improvement of performance in professional athletes ${ }^{5}$.

Furthermore, a 2013 study looked at the potential effects of enhancing caffeine performance compared to coffee, concluding that caffeine consumption in coffee ( $5 \mathrm{mg} / \mathrm{kg}$ body weight) and supplement ( $5 \mathrm{mg} / \mathrm{kg}$ body weight) ) one hour before the exercises can improve the endurance performance ${ }^{6}$.

A 2016 analysis concluded that there are indications that using coffee (as opposed to caffeine) as an ergogenic stimulant can improve cycling performance and long-distance running ${ }^{7}$.The authors suggested that the coffee intake that provides between $3-8.1 \mathrm{mg} / \mathrm{kg}$ of caffeine can be used as a safe alternative to anhydrous caffeine to improve endurance performance ${ }^{7}$.

Another study, conducted in 2017 on male athletes, suggests that 60 minutes after ingesting $0.09 \mathrm{~g} / \mathrm{kg}$ of caffeinated coffee, the performance in a onemile race was improved by $1.9 \%$ and $1.3 \%$ compared to a placebo and decaffeinated coffee, respectively, among professional male athletes ${ }^{8}$.

A 2017 meta-analysis suggests that caffeine intake had a suppressive effect on perceived exertion and no effect on the pulse, respiration, or $\dot{\mathrm{VO}}_{2}$ measurements. The authors suggested that while the positive effects of the additional intake of caffeine in performing high intensity sustained exercises are accepted, the mechanisms to explain this response remain unresolved ${ }^{9}$.

A small number of studies have considered a potential ergogenic effect of the low and very low intake of caffeine subsequently consumed in case of prolonged exercises. Low caffeine intake ( $200 \mathrm{mg}$ ) has been shown to improve alertness, as well as cognitive processes during and after strenuous exercise, but there is a lack of information on the potential effects on sprint activity. high intensity and tension. Because the response to caffeine consumption is variable, athletes should determine individually whether ingesting a lower amount of caffeine before and / or after workouts and competitions is ergogenic ${ }^{10}$.

\section{Caffeine and muscle pain}

In 2009, a research paper presented the effects of caffeine on muscle pain during a 30-minute high-intensity cycling session. Ingestion of caffeine (5 mg / kg body weight) had a statistically significant effect by reducing the intensity of reported muscle pain, the effect being greater in the group of regular users of low caffeine $^{11}$.

A 2011 study examined the effect of caffeine on foot pain and the perceived stress ratio, although caffeine intake improved various performance measures. The authors concluded that the suggestion is plausible that the subjects performed better with a similar level of pain and effort after consuming $5 \mathrm{mg} / \mathrm{kg}$ of caffeine compared to a placebo ${ }^{12}$. 


\section{Caffeine and performance in high-intensity short-term exercises (anaerobic)}

Research suggests that this substance may have benefits in certain highintensity exercises of short duration and under certain conditions ${ }^{13-15}$.

An analysis from 2009 that examined the effects of caffeine on performance in anaerobic exercise took into account 29 studies, finding that 17 of them showed that the substance has a significant effect ${ }^{13}$. It was also observed that there is a significant difference in the level of studies. Some factors from different studies were noted as possible explanations for this variation: trained participants vs. untrained, participants who used to consume caffeine vs. participants who did not use caffeine, organisms that metabolize slower vs. faster caffeine among participants, different dosage regimens (fixed amount of caffeine vs. mg per kg of body weight) and types different tests.

The results of a meta-analysis conducted in 2017 indicated a significant difference between placebo-based and caffeine-based clinical trials on average strength and maximum strength produced on an ergometer bike. This meta-analysis complements the current body of research that suggests that the ingestion of caffeine may enhance the components of anaerobic performance ${ }^{15}$.

Overall results suggest that caffeine intake may have benefits in highintensity short-term exercises, especially under certain conditions, such as among trained athletes who have refrained from consuming caffeine prior to strength sports events and sports. after moderate caffeine consumption ${ }^{13-15}$.

\section{Caffeine and carbohydrates}

A paper from 2010 analyzed the effect of caffeine $(3.7 \mathrm{mg} / \mathrm{kg}$ body weight) consumed in addition to a carbohydrate-electrolyte supplement in the performance of a simulated football game ${ }^{16}$. The authors found that the group that consumed caffeine performed better and improved performance in running speed and jumps over a short distance, compared to the group that did not use caffeine ${ }^{16}$. A 2011 analysis of the research suggests that ingesting carbohydrates with caffeine offers a significant but reduced improvement in endurance performance compared to carbohydrate-only administration ${ }^{17}$. However, the magnitude of the performance benefit that caffeine offers was lower when carbohydrates were added compared to placebo ${ }^{17}$.

\section{The effects of caffeine in the short term}

A paper from $2010^{18}$ reported that caffeine intake of $6 \mathrm{mg} / \mathrm{kg}$ body weight in trained women resulted in performance improvement in an "all in one" type test but not in a repeated test ${ }^{18}$.

Another paper ${ }^{19}$ tested two different types of caffeine intake $(2 \mathrm{mg} / \mathrm{kg}$ body weight and $5 \mathrm{mg} / \mathrm{kg}$ body weight) among active participants ${ }^{19}$. Ingestion of higher, and not lower, amounts of caffeine resulted in improved performance at 
knee extension/flexion type exercises. This effect disappeared in the second round, which means that any benefits of caffeine manifested only in the short term ${ }^{19}$.

\section{Caffeine and fluid balance during physical activity}

Fluid balance is especially important for athletes, as dehydration is always a concern associated with poor performance. Caffeine may exert a short-term diuretic effect, but research suggests that this effect does not counteract the effects of liquid intake from coffee consumption ${ }^{24-31}$. Moderate consumption of caffeinated coffee can help maintain a proper balance of fluids ${ }^{24-31}$.

A comprehensive analysis concluded that the daily intake of $300 \mathrm{mg}$ of caffeine (the amount determined in approximately 3 normal cups of coffee) induces only a weak, short-term diuretic effect similar to water, without having a significant effect on the overall balance of liquids ${ }^{24}$. The authors mentioned that there is no evidence that this substance is harmful during exercises performed in hot climatic conditions when fluid losses are maximum ${ }^{24}$. Later, the study confirmed that the claims that suggest avoiding caffeinated beverages before and during the exercises are unfounded ${ }^{24}$.

A 2014 meta-analysis considered the role of caffeine in the balance of fluids in adults during rest and exercise and concluded that although it produces a minor diuretic effect, it was canceled by exercise. The authors also suggested that concerns about unwanted fluid loss associated with caffeine intake are unfounded, especially when ingestion precedes exercise ${ }^{29}$.

Another study published in 2014 found that there are no significant differences in the measurements of the degree of hydration between those who consumed coffee or those who consumed water, concluding that moderate consumption of coffee by men who regularly consume coffee has similar hydration qualities. with water ${ }^{30}$.

In 2004, the International Olympic Committee (IOC) officially removed caffeine from the list of banned substances, stating that previous findings that the short-term diuretic effect of caffeine may affect physical performance are unfounded.

\section{Potential mechanism}

The ergogenic effects of caffeine were considered at one time to be explained by the ability of caffeine to stimulate the oxidation of free fatty acids and, consequently, to maintain muscle glycogen ${ }^{34}$, other similar mechanisms are currently being investigated.

\section{Endurance exercises}

Any suggested improvements in sports performance are more solid when it comes to endurance sports. Research and analysis have concluded that, primarily, caffeine affects endurance exercise through its antagonistic effect on adenosine 
receptors in the brain, for example through a process that leads to increased adrenaline production, which stimulates energy production and improves blood flow to muscles and heart ${ }^{6}$. Caffeine modulates central fatigue and influences perceived effort, perceived pain, and vigor, all of which can lead to improved performance ${ }^{6}$.

\section{Anaerobic exercises, of short duration}

For short-term anaerobic exercises, fatty acid oxidation and glycogen storage are not a realistic model for the mechanism behind performance improvement, for example, periods do not correspond. An analysis of 2009 on anaerobic mechanisms of action addressed current options and showed that it is not yet clear how caffeine improves performance in high-intensity exercises and short duration ${ }^{13}$.

Some of the domains investigated include lactic acid, blood glucose, potassium-for peripheral mechanisms; caffeine as an adenosine antagonist, pain perception and perceived effort-for the central mechanism. This central caffeine model is the most promising to date.

It is interesting that models of action mechanisms designed to explain the benefits of caffeine for performance in both types of exercise - endurance and high intensity and short duration - seem to be heading in the same direction ${ }^{17-19}$. As a consequence, the recommendations dedicated to athletes increasingly show similarities ${ }^{17-19}$.

\section{Genetic variability}

The researchers suggested that the impact of caffeine consumption on performance may be different. A clearer understanding of the factors that generate inter-individual variations can facilitate the customization of caffeine intake indications, specific to a person's biology, history, and competitive situation ${ }^{35}$.

\section{Conclusions}

Despite the beneficial properties of caffeine for increasing physical and mental energy, athletes should be cautious because:

1. For those who have not consumed caffeine, caffeine can have diuretic effects. This effect usually manifests until the body develops a significant tolerance to caffeine. Recent research has shown that consuming less than $300 \mathrm{mg}$ of caffeine per day does not have diuretic effects on inexperienced consumers or those already familiar with caffeine ${ }^{36}$.

2. The first time consumption of caffeine at high temperatures can cause or potentiate dehydration, leading to thermal shock.

3. Excessive caffeine consumption above the level required to increase energy can lead to caffeine. Caffeine is a state of addiction and can have the opposite effect, of decreasing mental and physical performance. 
4. Among the side effects due to the excessive consumption of caffeine include nervousness, anxiety, irritability, muscle spasms, insomnia, and headaches. Caffeine tolerance settles quickly in routine consumers and causes them to consume increasing doses to experience the same effects.

5. Cessation of caffeine consumption may lead to withdrawal. Symptoms of withdrawal include headaches, irritability, low concentration power, mild stomach pains, symptoms that can develop within the next 12 to 24 hours and can last up to 96 hours $^{36}$.

6. Excessive caffeine intake during workouts can lead to gastrointestinal discomfort (hyperacidity).

According to recent studies, caffeine intake before a competition or training may increase the risk of muscle damage. However, according to other studies, caffeine consumption can reduce post-workout muscle pain by up to $48 \%$ (especially for beginners). Caffeine users also seem to have a lower perception of effort during training, both speed and endurance, compared to those who do not use caffeine $^{36}$.

\section{REFERENCES}

1. Burke L.M. (2008) Caffeine and sports performance. Appl Physiol Nutr Metab, 33(6):1319-34. doi: 10.1139/H08-130.

2. Goldstein E.R. et al. (2010) Position Stand: caffeine and performance, $J$ In Soc Sports Nutr, 27;7(1):5.

3. Ganio M. S. et al. (2009) Effect of Caffeine on Sport-Specific Endurance Performance: A Systematic Review. J Strength Cond Res, 23(1):315-24.

4. Irwin C. et al. (2011) Caffeine withdrawal and high-intensity endurance cycling performance. J Sports Sci, Mar 29(5):509-15.

5. Desbrow B. et al. (2012) The effects of different doses of caffeine in endurance cycling time trial performance, J Sports Sci., 30(2):115-20.

6. Hodgson A.B. et al. (2013) The metabolic and performance effects of caffeine compared to coffee during endurance exercise. PLoS One, 8(4):e59561.

7. Higgins S. et al. (2016) The effects of pre-exercise caffeinated coffee ingestion on endurance performance. Int J Sport Nutr Exerc Metab, 26(3):221-39.

8. Clarke N. et al. (2017) Coffee ingestion enhances one-mile running race performance. Int J Sports Physiol Perform, published online ahead of print.

9. Glaister M., Gissane C. (2017) Caffeine and physiological responses to submaximal exercise: a meta-analysis. Int J Sports Physiol Perform, 5:1-23.

10. Spriet L.L. (2014) Exercise and sport performance with low doses of caffeine. Sports Med, 44(2):175-184.

11. Gliottoni R.C. et al. (2009) Effect of Caffeine on Quadricep Pain During Acute Cycling Exercises in Low Versus High Caffeine Consumers. J. Sport Nutr Exer Metab, 19:150-161. 
12. Astorino T.A. et al. (2011) Effect of caffeine intake on pain perception during high-intensity exercise. Int J Sport Nutr Exerc Metab, 21(1):27-32.

13. Davis J.K. et al. (2009) Caffeine and anaerobic performance - ergogenic value and mechanisms of action. Sports Med, 39:813-832.

14. Astorino T.A. et al. (2009) Efficacy of acute caffeine ingestion for short-term high-intensity exercise performance: a systematic review. J Strength Cond Res, 24(1):257-65.

15. Grgic J. (2017) Caffeine ingestion enhances Wingate performance: a metaanalysis. Eur J Sport Sci, 31:1-7.

16. Gant N. et al. (2010) The influence of caffeine and carbohydrate coingestion on simulated soccer performance. Intern. J. Sport Nutr Execr Metab, 20:191-197.

17. Conger S.A. et al. (2011) Does caffeine added to carbohydrate provide additional ergogenic benefit for endurance? Int J Sport Nutr Exerc Metab, 21(1):71-84.

18. Goldstein E.R. et al. (2010) Caffeine enhanced upper body strength in resistance trained women. Soc. Sports Nutrition, 7:18.

19. Astorino T.A. et al. (2010) Effect of two doses of caffeine on muscular function during isokinetic exercise. Med Sci Sport Execr, 42(12):2205-10.

20. EFSA Panel on Dietetic Products, Nutrition and Allergies (NDA) (2011) Scientific Opinion on the substantiation of health claims related to caffeine and increase in physical performance during short-term high-intensity exercise (ID $737,1486,1489$ ), increase in endurance performance (ID 737, 1486), increase in endurance capacity (ID 1488) and reduction in the rated perceived exertion/effort during exercise (ID 1488, 1490) pursuant to Article 13(1) of Regulation (EC) No 1924/2006. EFSA Journal, 9(4): 2053.

21. Astorino T.A. et al. (2012) Effect of caffeine on RPE and perceptions of pain, arousal, and pleasure/displeasure during a cycling time trial in endurance trained and active men. Physiol Behav, 106(2):211-7.

22. Laurence G. et al. (2012) Effects of caffeine on time trial performance in sedentary men. J Sports Sci, 30(12):1235-40.

23. EFSA Panel on Dietetic Products, Nutrition and Allergies (NDA) (2011) Scientific Opinion on the substantiation of health claims related to water and maintenance of normal physical and cognitive functions (ID 1102, 1209, 1294, 1331), maintenance of normal thermoregulation (ID 1208) and "basic requirement of all living things" (ID 1207) pursuant to Article 13(1) of Regulation (EC) No 1924/2006. EFSA Journal, 9(4):2075.

24. Maughan R.J. et al (2003) Caffeine ingestion and fluid balance: a review. $J$ Hum Nutr Diet, 16(6):411-20.

25. Neuhauser-Berthold M. et al (1997) Coffee consumption and total body water homeostasis as measured by fluid balance and bioelectrical impedance analysis. Ann Nutr Metab, 41(1):29-36.

26. Grandjean A.C. et al (2000) The effect of caffeinated, non caffeinated, caloric and non caloric beverages on hydration. J Am Coll Nutr, 19(5):591-600. 
27. Armstrong L.E. et al (2005) Fluid, electrolyte, renal indices of hydration during 11 days of controlled caffeine consumption. Int J Sport Nutr Exerc Metab, 15(3):252-65.

28. Silva A. M. et al (2013) Total body water and its compartments are not affected by ingesting a moderate dose of caffeine in healthy young adult males. Appl Physiol Nutr Metab, 38:626-632.

29. Zhang Y. et al. (2014) Caffeine and diuresis during rest and exercise: A metaanalysis. J Sci Med Sport, S1440-2440(14).

30. Killer S. C. et al. (2014) No Evidence of Dehydration with Moderate Daily Coffee Intake: A Counterbalanced Cross-Over Study in a Free-Living Population. PLoS ONE, 9(1): e84154.

31. Armstrong L.E. (2002). Caffeine, body fluid-electrolyte balance, and exercise performance. Int J Sport Nutr and Exerc Metab., 12:205-222.

32. European Food Safety Authority (2015) Scientific Opinion on the Safety of Caffeine. EFSA, Palma, Italy. EFSA Journal, 13(5):4102.

33. Costill, D. L. et al. (1978) Effects of caffeine ingestion on metabolism and exercise performance. Medicine and Science in Sport and Exercise, 10:155-158.

34. Tarnopolsky M.A. (1994) Caffeine and Endurance Performance. Sports Med, 18(2):109-25.

35. Pickering C. and Kiely K. (2017) Are the current guidelines on caffeine use in sport optimal for everyone? Inter-individual variation in caffeine ergogenicity and a move towards personalised sports nutrition. Sports Med, published online ahead of print.

36. http://www.desprecafea.info/cafeaua-si-performanta-sportiva-2/

37. https://nutritionist.info.ro/2011/10/03/cafeina-si-efectul-ei-asupra-performanteisportive/ 